\title{
Analysis of Academic Social Platform Based on Collaborative Innovation Mechanism
}

\author{
Zhou Jiawen \\ Nanjing University of Science and Technology, Nanjing China
}

\begin{abstract}
Keywords: Collaborative innovation; College; Academic social; Platform
\end{abstract}
Abstract. In the recent years, the higher education of our country has flourished and all the colleges have gradually widen the range of schools, and college financial management has gradually explored the new development way on the original basis. College financial management is still the core of all the college basic work of our country at any time, but how to integrate the innovative element into the collaborative innovation mechanism on the special new period and analyze the innovative idea of collaborative innovation mechanism under the new period is the specific aim. Innovating collaborative innovation mechanism research is the kind of hard and valuable task under the new period. This research analyzes the academic social platform under the scientific and reasonable collaborative innovation mechanism aiming at the developing situation of large part of academic social platform, whose main position is college academic social platform of our country and background is collaborative innovation mechanism in the new era. Furthermore, it makes the collaborative innovation mechanism gradually adapt the special environment of this new period, and establishes the good beacon direction for the academic social platform of our country.

\section{Preface}

This research deeply analyzes the advantages of collaborative innovation mechanism development in the new era and does the specific research with the existing problems and current situation in the present academic social platform construction, with the main theme environment of the academic social platform of our country. And it analyzes all kinds of existing problems in the academic social platform under the collaborative innovation mechanism of the new era from many aspects, and suggestively puts forward the specific solving method, so that the strategic and systematic thinking can be finished to establish the innovative research thinking model. In fact, collaborative innovation mechanism belongs to the mechanism of sharing information and knowledge within the group organization, aiming at collaboration and emphasizing at innovation, whose characteristic is that every single and independent innovative subject has the same target and power. People can use the same technical method to do the multiple communication and diversified collaboration. In the process, it is the priority among priorities of our work that making the collaborative innovation mechanism gradually adapt to the special environment of the new period, at the same time, it establishes the good beacon direction for the academic social platform of our country, leading to the development and expansion of a series of collaborative innovation mechanism research ideas.

\section{The academic social platform of our country}

Academic social platform. In the current academic social platform construction of our country, the academic social platform regarding the college as its position is the one that has the largest number, the biggest influence and the most extensive covering people, which not only closely connects with other academic platforms nationwide, for example, other high-end academic social platform the scientific research institutions, the famous circle of politics and business and new science and technology field. Thus, it is obvious to know the significance of college academic social platform in it. But to some extent, academic social platform can also be thought as the people with high education background study the interesting topics with many people through the academic research tools and skills where the aim is to strengthen the "academy", which not only emphasizes the strong purpose, 
but has a higher requirement to the timeliness of communication. In addition, once such pure academic social relationship formed, it is easy to maintain and develop.

The existing problem of academic social platform construction. There exists many problems to be solved in the academic social platform construction, but in this process, the first one to be solved is that some academic social platforms are not purely doing academy and always mingle with the relation of interest. What's more, some researchers don't study hard enough, namely, they can't understand what the real meaning of the motivation and soul of doing academy, just executing and repeating the former topic and task stiffly without any innovation sense. Or there are some academic social platform that have too strong commercial purpose, and some researchers have not strong protection sense of scientific patent of themselves, so they always can make some people with other purposes profit from them, which is truly what we should abandon. Therefore, under certain circumstances, we should focus on the standardization of academic social platform construction to make the related work transparent. Meanwhile, we should strengthen the relation among all large-scale academic platforms, establish the review committee of academic social platform construction and set the specific departments and people to do the relevant reviewing work in order to make the entire work sequencing, scientific and systematic and get rid of some traditional disadvantages. In the recent years, the amount and scale of academic social platform has gradually expanded, but the problems brought by the expansion are endless, and the standard of setting academic social platform also has been questioned by all sectors of society. Thus, it is the other problem to be solved that how to guarantee the quality in the platform construction. However, the problem with a deeper level brought by these problems is that they make the academic platform construction in the new era not innovative, namely, staying still and not moving at all, so the problem that is in the greatest need to be solved is that solving these problems and putting forward the solving methods.

\section{The development idea of academic social platform under the collaborative innovation mechanism}

There exists the very important relationship between the construction work of collaborative innovation mechanism and the normal office and operating of academic social platform. However, some problems have appeared with the great breakthrough of the construction work of collaborative innovation mechanism in the new era. Same, they also cause much needless trouble with the ordinary work of academic social platform. So, it is the most pressing key task to be solved in the long term that how to specifically boost the construction work of collaborative innovation mechanism in the current big new-era environment and how to make full use of the special environment advantage in the new era, avoiding the occurrence of problems in the meantime. In the recent years, academic social platform has expanded its amount and size little by little, but how to establish the perfect collaborative innovation mechanism also is impending, so the following few points of development ideas are worthy of being carefully thought by the constructor and manager of academic platform.

Strengthen the connection among the academic social platforms. In the new period, what should be noticed is how to closely connect with all the academic social platforms, how to find the existing problems in the research collaborative innovation mechanism construction comprehensively, specifically and systematically, and find the source of this problem, the reason of its occurrence and the way to avoid the next happening. Thus, we should pay attention to the standardization of academic social platform construction under some circumstances to make the relevant work transparent and strengthen the connection among all the major academic platforms at the same time. In addition, we should focus on establish the review committee of academic platform construction and found the specific departments and people to do the related reviewing work, which can make the entire work procedure sequencing, scientific and systematic, and get rid of some traditional disadvantages. Meanwhile, we are supposed to establish and improve the relevant assessment mechanism system, guarantee the smooth development of academic communication platform work, confirm the main direction of all the work and make the work thought further clear furthermore. On 
the other hand, we also can put forward the specific and feasible innovation measures to ensure the synchronous development of thought and measure, which is the beacon direction of future work.

Positively boost the collaborative innovation mechanism construction. Make full use of the advancement of academic platform management method being the center to positively boost the collaborative innovation mechanism construction, and all the academic social platforms should make full use of their own power, strengthen the information construction of related group organization and enhance the efficiency and progress of all the work following with the time tide. Lots of people study the topic all the people are interested together, which aims to strengthen the "academy" and not only emphasizes the strong purpose but also needs the better communication timeliness requirement. What's more, once this kind of pure academic social relationship formed, it is easy to maintain and develop. Thus, it is the other problem to be solved that how to guarantee the quality in the platform construction. However, the problem with a deeper level brought by these problems is that they upset the enthusiasm of researchers and the innovation sense of staff becomes not obvious. Therefore, it is the key point that cultivating the collaborative innovation sense of members and improving the academic social platform construction.

Establish the perfect management regulation of academic social platform. In the college financial management work, the affiliated unit of each social platform should make the analysis on the relevant image according to the practical situation to make the academic social platform management regulation with normative system. The academic contest of our country has been more and more fierce in the new period, so we should not only enhance the entire quality of entire platform, but also take the corresponding responsibility and make the management responsibility implement into each related responsible person, always regarding the academic social platform management as the first task of every unit development. In addition, strengthening the management training of staff, supervising and urging the relevant person to continually study the related knowledge and doing the related course well can make the tough problems easily solved. On the other hand, the manager and constructor of social platform should finish the transformation of different roles, and accomplish the gradual transformation of traditional academic work in the meantime. The total academic circle should make full use of its own platform and strengthen the efficiency and process of all the work.

\section{Conclusion}

Overall, based on the special environment of the new period, this paper studies and analyzes a series of innovative collaborative innovation mechanism construction aiming at the current situation of most part of academic social platform, furthermore, it develops varieties of colorful project research and analyzes the existing problems of collaborative innovation mechanism construction of new period from many aspects. In addition, this paper suggestively puts forward the specific solving method to finish the strategic and systematic thinking and establish the innovation research thinking model. The writer deeply analyzes the advantage that environment of new period brings to the academic social platform construction, and makes the specific research with the existing problem and current situation of construction in the meantime. Collaborative innovation mechanism indeed is the mechanism where the group can communicate and share information, aiming at collaboration and emphasizing at innovation. And its characteristic is that every single and independent innovation subject possesses the same purpose and motivation. In the new era, innovation collaboration innovative mechanism is a hard and valuable task. This research analyzes the academic social platform under the scientific and reasonable collaborative innovation mechanism aiming at the developing situation of large part of academic social platform, whose main position is college academic social platform of our country and background is collaborative innovation mechanism in the new era. Furthermore, it makes the collaborative innovation mechanism gradually adapt the special environment of this new period, and establishes the good beacon direction for the academic social platform of our country. However, because the academic platform construction also exists lots of problems, and a series of risk sometimes makes work hesitate, so if the relevant function approval department cannot have the right attitude and take the clear attitude, even cannot make a elaborate 
collaborative innovation mechanism innovation scheme, then it is very possible to make the risk of academic level increasing and some potential problems will break out at any time and make all the work of the total platform severely affected. This research deeply analyzes the advantages of collaborative innovation mechanism development in the new era and does the specific research with the existing problems and current situation in the present academic social platform construction, with the main theme environment of the academic social platform of our country. And it analyzes all kinds of existing problems in the academic social platform under the collaborative innovation mechanism of the new era from many aspects, and suggestively puts forward the specific solving method, so that the strategic and systematic thinking can be finished to establish the innovative research thinking model. Whatever, traditional collaborative innovation mechanism construction work has been under the pressure from all aspects in the new period, so how to successfully transform the collaborative innovation mechanism construction work is the prominent problem needing to be solved at present, in the meantime, which also establish the good cultivation direction for the relevant department leading to the development of a series of academic social platform construction research idea under the collaborative innovation mechanism.

\section{References}

[1] Yu Yang, Song Liu. The innovation and practice research of education and science basic level under the "collaborative innovation mechanism" $[\mathrm{J}]$. Culture and education material, 2014(29):160-161.

[2] Yu Hong. The research of effect mechanism of college collaborative innovation on the academic output quality - the citation analysis based on the Sino-US high-level university[J]. Educational Research of Tsinghua University, 2016, 37(5).

[3] Xuewen Zhang. The collaborative innovation of industry, college and research from the open scientific eagle — system logic, contract management, and social welfare[J]. Scientific research, 2013, 31(4):617-622.

[4] Xiaojuan Shi, Jiwen Sun. The study of the content, reference experience and innovation mechanism of the collaborative innovation of philosophy and society science in the college[J]. Scientific management research,2013, v.33;No.296(22):92-95.

[5] Xiongyi Li. The study of collaborative innovation mechanism based on the analysis of financial service risk and benefit[J]. The 17th academic seminar of cross-strait information management development and strategy,2013.

[6] Fan Wang. Collaborative innovation: the reform exploration of college basic-level academic organization and operation mechanism[J]. Education circle: research of higher education,2015(7):126-126.

[7] Xiaojun Tang, Ying Lu, Na Liu, etc. The research and practice of collaborative innovation mechanism that together constructs and shares the excellent resource and course under the background of society transformation[C]// The abstract work of excellent papers with the third prize in the academic annual meeting of Liaoning province higher education journal. 2014.

[8] Yanfang Lv. The research of interdisciplinary talent training mode mechanism of graduate students under the concept of collaborative innovation[J]. Scientific horizon, 2016(7):78-79. 